\title{
Wide-Line Proton Magnetic Resonance Studies of Deuterated Poly $(\gamma$-benzyl glutamate). Verification of Previous Interpretation for Side Chain Motion
}

\author{
Akihiro TsuTsumI,* Saburo ANZAI, and Kunio HiKICHI \\ Department of Polymer Science, Faculty of Science, \\ Hokkaido University, Kita-ku, Sapporo 060, Japan
}

(Received November 9, 1982)

\begin{abstract}
The wide-line proton magnetic resonance of poly( $\gamma$-benzyl glutamate) (PBG) was studied using two deuterated samples. One was deuterated at the phenyl groups, PBG- $d_{5}$, and the other, at the benzyl groups, PBG- $d_{7}$. The temperature dependence of the line width and the second moment was compared with that for PBG. It was found that the use of these deuterated samples made it possible to identify the particular part of the side chain participating in the molecular motion. The results indicated that in PBG, the side chain motion is excited first at the end group and then propagates to the backbone with rise in temperature, in agreement with our previous interpretation. An attempt was made to decompose the second moment of PBG into several components.
\end{abstract}

KEY WORDS Poly $(\gamma$-benzyl glutamate) / Deuteration / Side Chain Motion /
NMR /

Many studies on the physical properties of $\alpha$ helical poly(amino acid)s in the solid state have shown that the side chain undergoes rapid motion above a certain temperature, while the backbone maintains a rigid structure. ${ }^{1-9}$ Thus, it is important to know the mechanism of the side chain motion in order to understand the physical properties of poly(amino acid)s. It is well established that wideline nuclear magnetic resonance (NMR) measurements give useful information on the molecular motion occuring in solid polymers. We have already employed this technique for poly(amino acid)s including poly $\left(\gamma\right.$-benzyl glutamate) $(\mathrm{PBG})^{2,3}$ with the chemical structure

$$
\begin{aligned}
& t^{\mathrm{H}}-\underset{1 \alpha}{\mathrm{C}} \mathrm{H}-\stackrel{\mathrm{O}}{\mathrm{C}}^{\prime}-f_{\mathrm{n}}
\end{aligned}
$$

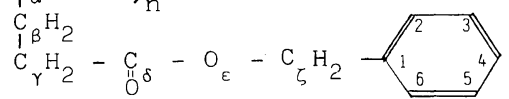

In this work, we again carried out NMR measurements on PBG to obtain additional support for our previous interpretation of the side chain motion, ${ }^{2,3}$ using PBG- $d_{5}$ and PBG- $d_{7}$ in which protons in the phenyl and benzyl groups are deuterated, respectively.

\section{EXPERIMENTAL}

\section{Sample Preparation}

Poly $\left(\gamma\right.$-benzyl- $d_{5}$ D-glutamate) (PBG- $\left.d_{5}\right)$. Poly $(\gamma-$ methyl D-glutamate) (PMDG) was obtained by polymerizing the $N$-carboxyanhydride (NCA) of $\gamma$ methyl D-glutamate in a mixture of dichloromethane and dioxane $\left(50: 50, \mathrm{vol}^{\%}\right)$ at room temperature using triethylamine as the initiator. PMDG was converted to PBG- $d_{5}$ by an ester exchange reaction at $60^{\circ} \mathrm{C}$ with benzyl alcohol- $d_{5}$ in dichloroethane containing $p$-toluene-sulfonic acid as a catalyst. The degree of exchange was $97 \%$, as determined from high resolution ${ }^{1} \mathrm{H}$ NMR spectrum in $\mathrm{CDCl}_{3}$-TFA (trifluoroacetic acid) $(60: 40$, $\mathrm{vol}^{\circ} \%$ ).

Poly ( $\gamma$-benzyl- $d_{7}$ D-glutamate) (PBG- $\left.d_{7}\right)$. DGlutamic acid $(12 \mathrm{~g})$ was mixed with $60 \%$ sulfuric

* Present address: Department of Applied Physics, Faculty of Engineering, Hokkaido University Kita-ku, Sapporo 060, Japan. 
acid $(14 \mathrm{~g})$ and benzyl alcohol- $d_{7}(10 \mathrm{~g})$. This mixture was heated at $70^{\circ} \mathrm{C}$ to obtain a clear solution. Water in the solution was allowed to vaporize at $70^{\circ} \mathrm{C}$. The concentrated solution was put into $52 \mathrm{ml}$ cold water containing $14 \mathrm{~g}$ of sodium bicarbonate. The precipitate was filtered, washed with cold water, and dried in vacuo. The purification was made by recrystallizing from hot water. Finally, $15 \mathrm{~g}$ of $\gamma$-benzyl- $d_{7}$ D-glutamate was obtained. This compound was then converted to an NCA in dioxane containing phosgen $\mathrm{COCl}_{2}$ (2 equiv mol). The purified NCA was allowed to polymerize in dioxane with triethylamine as the initiator to obtain PBG- $d_{7}$.

\section{NMR Measurements}

The samples for NMR measurements were washed with water, ethanol, and ether, and dried in vacuo. They were packed in glass tubes, evacuated at $130^{\circ} \mathrm{C}$ for 2 days to eliminate residual solvents and sealed. NMR derivative spectra were measured with a JEOL Pound-Watkins type NMR spectrometer operating at $12 \mathrm{MHz}$. The field modulation frequency was $30 \mathrm{~Hz}$, and the amplitude was adjusted to prevent modulation broadening. The temperature was varied from $-196^{\circ}$ to $150^{\circ} \mathrm{C}$.

\section{RESULTS AND DISCUSSION}

Figure 1 shows plots of the line width between the maximum and minimum of the derivative spectrum against temperature. A gradual line narrowing appears at $-100^{\circ} \mathrm{C}$ for PBG- $d_{5}$, and in the region of room temperature, an abrupt narrowing occurs for both samples. Figure 2 illustrates the temperature dependence of the second moment. It can be seen that the second moment decreases in the same temperature region as the line narrowing occurs.

In Figures 1 and 2, the dotted curves indicate the results for nondeuterated PBG. The gradual and abrupt narrowings below and near room temperature for PBG were interpreted as resulting from small-scale motion of the benzyl group and largescale motion of the entire side chain, respectively. ${ }^{1-3,6}$ This interpretation is given additional support by the present results for deuterated PBGs in the following way.

Figure 2 shows that the second moment for PBG decreases first in the temperature range from $-160^{\circ}$ to $-100^{\circ} \mathrm{C}$, but not in those for other samples, indicating that the motion of the phenyl group occurs in this temperature range. For PBG- $d_{5}$, the first decrease in the second moment is observed from $-100^{\circ}$ to $-50^{\circ} \mathrm{C}$. This decrease may be attributed to the motion of benzyl methylene groups $\left(\zeta-\mathrm{H}_{2}\right)$, since the second moment for PBG- $d_{7}$ remains unchanged in this temperature range. For PBG- $d_{7}$, the second moment maintains a constant value of 13 gauss $^{2}$ up to room temperature. Thus, it may be concluded that the motion of $\beta-\mathrm{H}_{2}$ and $\gamma-\mathrm{H}_{2}$

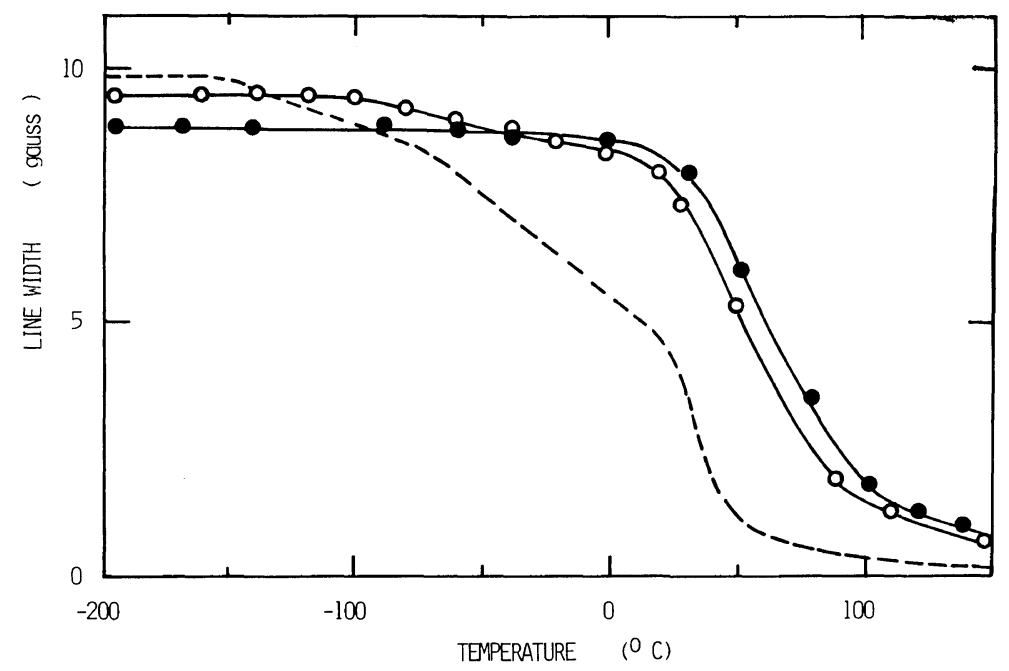

Figure 1. Temperature dependence of the line width for deuterated poly( $\gamma$-benzyl glutamate)s: $(\bigcirc)$, PBG- $d_{5}$; ( ), PBG- $d_{7}$; (----), PBG. 
NMR Studies of Deuterated PBG

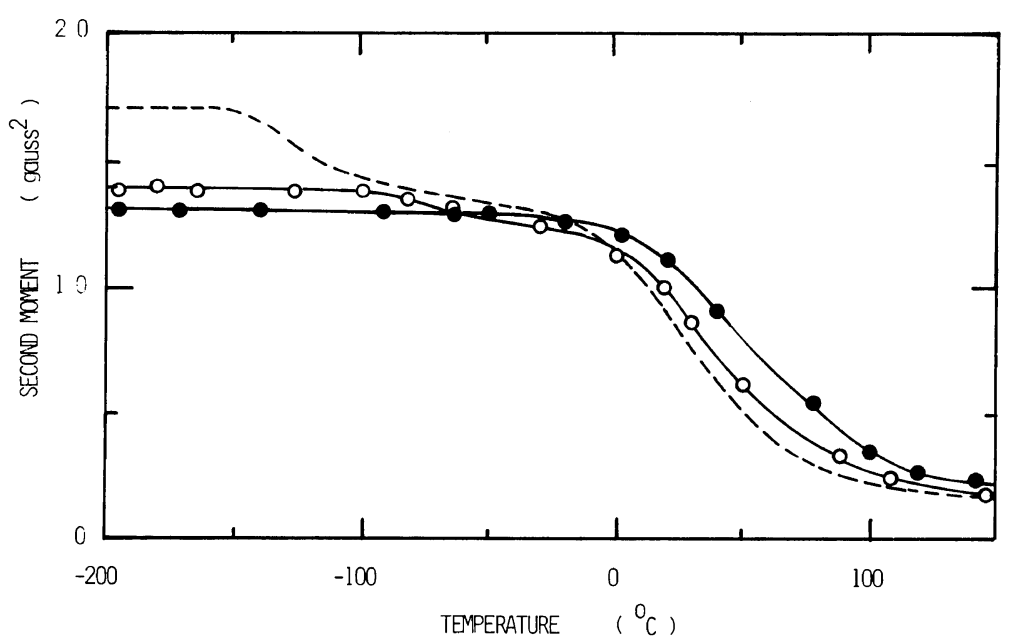

Figure 2. Temperature dependence of the second moment for deuterated poly $(\gamma$-benzyl glutamate)s: $(O)$, PBG- $d_{5}$; (९), PBG- $d_{7}$; (----), PBG.

does not occur below room temperature. Above room temperature, however, these protons undergo rapid motion, including the motion of polar carboxyl group. ${ }^{4,6}$ All of these results support our previous interpretation that the side chain motion in PBG is excited first at the end group and then proceeds to the backbone with increasing temperature. ${ }^{2-4,6}$

The second moment values at the lowest temperature were 17.0, 13.8, and 13.0 gauss $^{2}$ for PBG, PBG$d_{5}$, and PBG- $d_{7}$, respectively. These values allow the second moment for PBG to be decomposed into several contributions. The second moment of a ${ }^{1} \mathrm{H}$ NMR powder spectrum is given by Van Vleck ${ }^{10}$ as

$$
\left\langle\Delta H^{2}\right\rangle=\frac{3}{5} I(I+1)\left(\frac{\gamma h}{2 \pi}\right)^{2} \frac{1}{N} \sum_{i \neq j} r_{i j}^{-6}
$$

where $I$ is the nuclear spin of proton, $\gamma$ the magnetogyratic ratio, $h$ the Planck constant, $N$ the number of magnetic nuclei over which the sum is taken, $r_{i j}$ the length of the vector joining $i$ and $j$ nuclei. For PBG, if the monomeric unit containing 13 protons is taken as a structure unit, $N$ becomes $13 \times Q$ ( $\equiv N_{\mathrm{PBG}}$ ), where $Q$ is a constant determined by the number of structure units over which the magnetic interactions are taken into account. For PBG- $d_{5}$ and PBG- $d_{7}$, on the other hand, $N$ would be $8 \times Q$ and $6 \times Q$, respectively, and denoted as $N_{\mathrm{PBG}-d_{5}}$ and $N_{\mathrm{PBG}-d_{7}}$. In eq 1 , the sum should exclude phenyl and benzyl-protons for PBG- $d_{5}$ and PBG- $d_{7}$, respectively. Therefore, if the second moments for PBG
$\left(\left\langle\Delta H^{2}\right\rangle_{\mathrm{PBG}}\right)$, and deuterated PBGs $\left(\left\langle\Delta H^{2}\right\rangle_{\mathrm{PBG}-d_{5}}\right.$ and $\left.\left\langle\Delta H^{2}\right\rangle_{\mathrm{PBG}-d_{7}}\right)$ are known, the second moment contributions from phenyl $\left(\left\langle\Delta H^{2}\right\rangle_{\mathrm{P}}\right)$ and benzyl $\left(\left\langle\Delta H^{2}\right\rangle_{\mathrm{B}}\right)$ groups in PBG can be estimated as follows:

$$
\begin{aligned}
& \left\langle\Delta H^{2}\right\rangle_{\mathrm{P}}=\left\langle H^{2}\right\rangle_{\mathrm{PBG}}-R_{\mathrm{P}}\left\langle\Delta H^{2}\right\rangle_{\mathrm{PBG}-d_{5}} \\
& \left\langle\Delta H^{2}\right\rangle_{\mathrm{B}}=\left\langle H^{2}\right\rangle_{\mathrm{PBG}}-R_{\mathrm{B}}\left\langle\Delta H^{2}\right\rangle_{\mathrm{PBG}-d_{7}}
\end{aligned}
$$

where

$$
\begin{aligned}
& R_{\mathrm{P}}=N_{\mathrm{PBG}-d_{5}} / N_{\mathrm{PBG}}=8 / 13 \\
& R_{\mathrm{B}}=N_{\mathrm{PBG}-d_{7}} / N_{\mathrm{PBG}}=6 / 13
\end{aligned}
$$

$\left\langle\Delta H^{2}\right\rangle_{\mathrm{P}}$ and $\left\langle\Delta H^{2}\right\rangle_{\mathrm{B}}$ were found to be 8.5 and 11.0 gauss $^{2}$, respectively. The contribution of $\zeta$-protons in the benzyl group, $\left\langle\Delta H^{2}\right\rangle_{\zeta}$, was calculated from the difference of these two values, giving 2.5 gauss $^{2}$. The total contribution of residual $N-, \alpha-, \beta$, and $\gamma-$ protons, $\left\langle\Delta H^{2}\right\rangle_{\mathrm{M}}$, was estimated from the result for PBG- $d_{7}$, that is, $\left\langle\Delta H^{2}\right\rangle_{\mathrm{M}}=\left\langle\Delta H^{2}\right\rangle_{\mathrm{PBG}-d_{7}} R_{\mathrm{B}}$, giving 6 gauss $^{2}$. These decompositions of the second moment were made at other temperatures. Figure 3 shows the temperature dependence of $\left\langle\Delta H^{2}\right\rangle_{\mathrm{M}}$, $\left\langle\Delta H^{2}\right\rangle_{\zeta}$, and $\left\langle\Delta H^{2}\right\rangle_{\mathbf{p}}$.

The second moments $\left\langle\Delta H^{2}\right\rangle_{\mathrm{M}},\left\langle\Delta H^{2}\right\rangle_{\zeta}$, and $\left\langle\Delta H^{2}\right\rangle_{\mathrm{P}}$ can be further decomposed into several contributions as shown in Table I. These contributions are expressed in terms of the interactions between pairs of groups $\mathrm{A}, \mathrm{B}$, and $\mathrm{C}$ which contain $N-, \alpha-, \beta$, and $\gamma$-protons, $\zeta$-protons, and phenyl- 


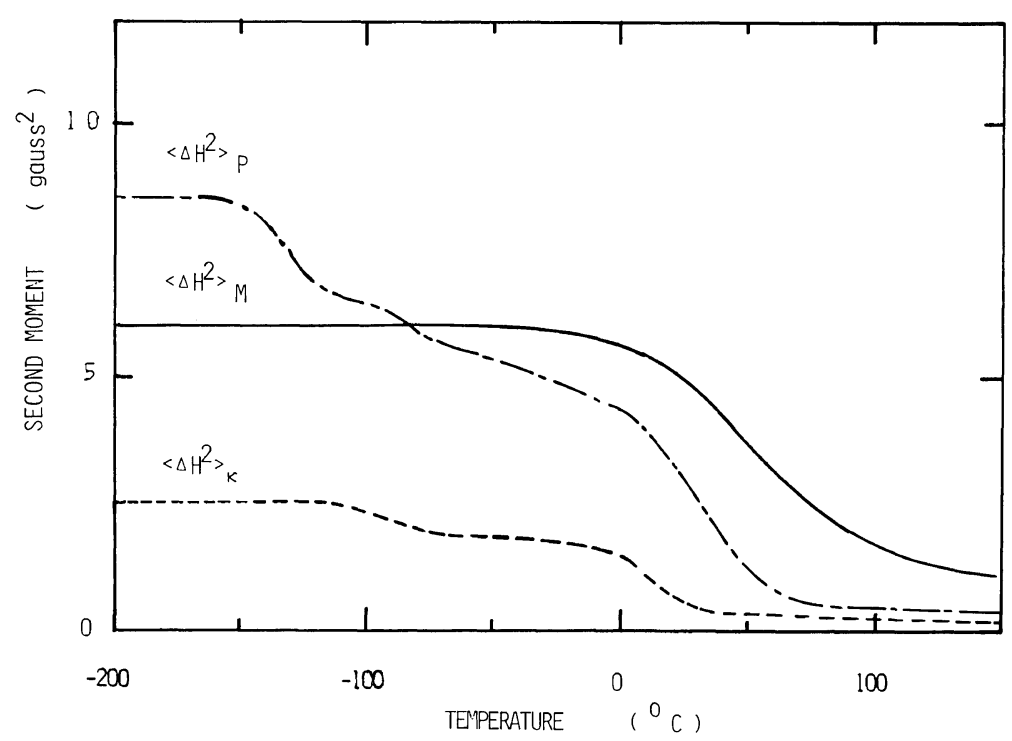

Figure 3. Temperature dependence of the decomposed second moment for poly $(\gamma$-benzyl glutamate): $(-),\left\langle\Delta H^{2}\right\rangle_{\mathrm{M}} ;(---),\left\langle\Delta H^{2}\right\rangle_{\zeta}(---),\left\langle\Delta H^{2}\right\rangle_{\mathrm{P}}$.

Table I. Components contributing to the second moment for $\operatorname{poly}(\gamma \text {-benzyl glutamate })^{\mathrm{a}}$

\begin{tabular}{|c|c|c|}
\hline $\mathrm{A}$ & B & $\mathrm{C}$ \\
\hline 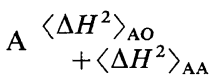 & $\left\langle\Delta H^{2}\right\rangle_{\mathrm{AB}}$ & $\left\langle\Delta H^{2}\right\rangle_{\mathrm{AC}}$ \\
\hline $\mathrm{B}\left\langle\Delta H^{2}\right\rangle_{\mathrm{BA}}$ & $\begin{array}{l}\left\langle\Delta H^{2}\right\rangle_{\mathrm{BO}} \\
\quad+\left\langle\Delta H^{2}\right\rangle_{\mathrm{BB}}\end{array}$ & $\left\langle\Delta H^{2}\right\rangle_{\mathrm{BC}}$ \\
\hline $\mathrm{C}\left\langle\Delta H^{2}\right\rangle_{\mathrm{CA}}$ & $\left\langle\Delta H^{2}\right\rangle_{\mathrm{CB}}$ & $\begin{array}{l}\left\langle\Delta H^{2}\right\rangle_{\mathrm{CO}} \\
\quad+\left\langle\Delta H^{2}\right\rangle_{\mathrm{CC}}\end{array}$ \\
\hline
\end{tabular}

a A, $N-, \alpha-, \beta$-, and $\gamma$-protons; B, $\zeta$-protons; C, phenyl protons.

protons, respectively. The second moments of the diagonal components in Table I are associated with the interactions within each group $\left(\left\langle\Delta H^{2}\right\rangle_{\mathrm{xo}}\right)$ and among the same groups $\left(\left\langle\Delta H^{2}\right\rangle_{\mathrm{XX}}\right)$ of different structure units, where $\mathrm{X}$ is $\mathrm{A}$ or B or C. The offdiagonal components are, on the other hand, due to the interactions among different groups $\left(\left\langle\Delta H^{2}\right\rangle_{\mathrm{XY}}\right)$. $\left\langle\Delta H^{2}\right\rangle_{\mathrm{Xx}}$ and $\left\langle\Delta H^{2}\right\rangle_{\mathrm{XY}}$ may be further decomposed into intra and intermolecular contributions. In terms of the symbols given in Table I, $\left\langle\Delta H^{2}\right\rangle_{\mathrm{M}}$, $\left\langle\Delta H^{2}\right\rangle_{\zeta}$, and $\left\langle\Delta H^{2}\right\rangle_{\mathbf{P}}$ are written as

$$
\left\langle\Delta H^{2}\right\rangle_{\mathrm{M}}=\left\langle\Delta H^{2}\right\rangle_{\mathrm{AO}}+S_{\mathrm{A}}
$$

$$
\begin{aligned}
\left\langle\Delta H^{2}\right\rangle_{\zeta} & =\left\langle\Delta H^{2}\right\rangle_{\mathrm{BO}}+S_{\zeta} \\
\left\langle\Delta H^{2}\right\rangle_{\mathrm{P}} & =\left\langle\Delta H^{2}\right\rangle_{\mathrm{CO}}+S_{\mathrm{P}} \\
\left\langle\Delta H^{2}\right\rangle_{\mathrm{PBG}} & =\left\langle\Delta H^{2}\right\rangle_{\mathrm{M}}+\left\langle\Delta H^{2}\right\rangle_{\zeta}+\left\langle\Delta H^{2}\right\rangle_{\mathrm{P}}
\end{aligned}
$$

where

$$
\begin{aligned}
S_{\mathrm{A}} & =\left\langle\Delta H^{2}\right\rangle_{\mathrm{AA}} \\
S_{\zeta} & =\left\langle\Delta H^{2}\right\rangle_{\mathrm{BB}}+\left\langle\Delta H^{2}\right\rangle_{\mathrm{BA}} \\
S_{\mathrm{P}} & =\left\langle\Delta H^{2}\right\rangle_{\mathrm{CC}}+\left\langle\Delta H^{2}\right\rangle_{\mathrm{CA}}+\left\langle\Delta H^{2}\right\rangle_{\mathrm{CB}}
\end{aligned}
$$

In the rigid state, isolated methylene and phenyl groups have the second moments of 11.1 and 2.1 gauss $^{2}$, respectively. Hence, $\left\langle\Delta H^{2}\right\rangle_{\mathrm{BO}}$ and $\left\langle\Delta H^{2}\right\rangle_{\mathrm{CO}}$ are estimated to be $11.1 \times(2 / 13)=1.7$ and $2.1 \times(5 /$ 13) $=0.8$ gauss $^{2}$, respectively. The major term in $\left\langle\Delta H^{2}\right\rangle_{\mathrm{P}}$ is, therefore, $S_{\mathrm{P}}$, which includes interactions with the surrounding groups. For $\left\langle\Delta H^{2}\right\rangle_{\mathrm{M}}$, the contribution only from $\beta$ - and $\gamma$-protons is 3.4 gauss $^{2}$, which gives a minimum value for $\left\langle\Delta H^{2}\right\rangle_{\mathrm{AO}}$. Theoretical calculations using the proposed conformations $^{11-13}$ for the side chain showed that $\left\langle\Delta H^{2}\right\rangle_{\mathrm{M}}$ of an isolated helix ranges from 6 to 8 gauss $^{2}$ which is comparable to the value of $\left\langle\Delta H^{2}\right\rangle_{\mathrm{M}}$ at $-196^{\circ} \mathrm{C}$. Therefore, it may be concluded that $S_{\mathrm{A}}$ arises primarily from intramolecular contributions.

Figure 3 shows that the decrease in the second moment for $\left\langle\Delta H^{2}\right\rangle_{\zeta}$ is 0.8 gauss $^{2}$ in the temperature 
range from $-120^{\circ}$ to $-70^{\circ} \mathrm{C}$. If the $\zeta$-methylene group rotates freely only about the $\mathrm{O}_{\varepsilon}-\mathrm{C}_{\zeta}$ bond, $\left\langle\Delta H^{2}\right\rangle_{\text {во }}$ should diminish by a value of $1.7 \times(3 /$ 4) $=1.3$ gauss $^{2}$, which is the minimum decrease in $\left\langle\Delta H^{2}\right\rangle_{\zeta}$ due to this motion and is larger than the observed value. It may thus be concluded that a rotational oscillation with a small amplitude about one or both of the $\mathrm{O}_{\varepsilon}-\mathrm{C}_{\zeta}$ and $\mathrm{C}_{\delta}-\mathrm{O}_{\varepsilon}$ bonds occurs from $-120^{\circ}$ to $-70^{\circ} \mathrm{C}$. The constant value above $-70^{\circ} \mathrm{C}$ implies that the rotational oscillation does not undergo further amplification up to room temperature. The temperature dependence of $\left\langle\Delta H^{2}\right\rangle_{\mathrm{P}}$ is very pronounced. This contribution dominates the second moment for PBG at lower temperature, but diminishes considerably near room temperature. Since $\left\langle\Delta H^{2}\right\rangle_{\mathrm{P}}$ is mainly due to $S_{\mathrm{P}}$, the temperature dependence describes that of the interactions of the phenyl groups with the same or different surrounding groups. The motion of a phenyl group should greatly affect the steric interaction with the surroundings, since this group is large in size and is strongly anisotropic and bulky in geometry. This may be a reason why $\left\langle\Delta H^{2}\right\rangle_{\mathrm{p}}$ shows a strong temperature dependence. It follows from the facts discussed above that the decrease in the temperature range from $-160^{\circ}$ to $-120^{\circ} \mathrm{C}$ and that from $-120^{\circ}$ to $-70^{\circ} \mathrm{C}$ is due to the rotational oscillation of phenyl groups about the $\mathrm{C}_{\zeta}-\mathrm{C}_{1}$ bond and a further increase in motional freedom due to the rotational oscillation about the $\mathrm{O}_{\varepsilon}-\mathrm{C}_{\zeta}$ bond, respectively. A further gradual decrease from $-70^{\circ} \mathrm{C}$ to room temperature is ascribed to a gradual increase in the amplitude of oscillation mainly about the $\mathrm{C}_{\zeta}-\mathrm{C}_{1}$ bond.
All of these findings again lend fair support to our previous explanation of the temperature dependence of the second moment for PBG., ${ }^{2,3}$

Acknowledgment. The authors are grateful to Professor T. Tokura in Hokkaido University for his valuable comments in regard to the preparation of the samples. This work was supported in part by a Grant-in-Aid of Scientific Research from the Ministry of Education, Science and Culture of Japan (Grant No. 57460190).

\section{REFERENCES}

1. J. A. Kail, J. A. Sauer, and A. E. Woodward, J. Phys. Chem., 66, 1292 (1963)

2. K. Hikichi, J. Phys. Soc. Jpn., 19, 2169 (1964).

3. A. Tsutsumi, Jpn. J. Appl. Phys., 9, 1125 (1970).

4. K. Hikichi, K. Saito, M. Kaneko, and J. Furuichi, J. Phys. Soc. Jpn., 19, 557 (1966).

5. A. Tsutsumi, K. Hikichi, M. Mitsumaki, and M. Kaneko, J. Phys. Soc. Jpn., 22, 1120 (1967).

6. A. Tsutsumi, K. Hikichi, T. Takahashi, Y. Yamashita, N. Matsushima, M. Kanke, and M. Kaneko, J. Macromol. Sci. Phys., B8, 413 (1973).

7. Y. Yamashita, A. Tsutsumi, K. Hikichi, and M. Kaneko, Polym. J., 8, 114 (1975).

8. Y. Yamashita, A. Tsutsumi, K. Hikichi, and M. Kaneko, Polym. J., 11, 241 (1979).

9. N. Sasaki, A. Tsutsumi, K. Hikichi, Y. Konishi, and M. Hatano, Polym. J., 11, 583 (1979).

10. Van Vleck, Phys. Rev., 74, 1168 (1948).

11. M. Tsuboi, J. Polym. Sci., 59, 139 (1962).

12. T. Ooi, R. A. Scott, G. Vandekooi, and H. A. Scheraga, J. Chem. Phys., 40, 4410 (1969).

13. A. Tanabe and Y. Ishida, J. Polym. Sci., 11, 1117 (1973). 